\title{
Exploring the construction of "Reference" triangle through unitarity
}

\author{
Monika Randhawa and Manmohan Gupta \\ Department of Physics, \\ Centre of Advanced Study in Physics, \\ Panjab University, Chandigarh- 160 014, India.
}

November 3, 2018

\begin{abstract}
Motivated by the possibility of the low value of $\sin 2 \beta$ in the measurements of BABAR and BELLE collaborations, we have explored the possibilty of construction of reference unitarity triangle using the unitarity of the CKM matrix, the existence of nonzero CP violating phase $\delta$ and the experimental values of the well known CKM elements, without involving any inputs from the processes which might include the new physics effects. The angles of the reference triangle are evaluated by finding $\delta$ through the Jarlskog's rephasing invariant parameter $J$. The present data and the unitarity of the CKM matrix give $\delta=50^{\circ} \pm 20^{\circ}$, which translates to $130^{\circ} \pm 20^{\circ}$ in the second quadrant. The corresponding range for $\sin 2 \beta$ is 0.21 to 0.88 . This range is broadly in agreement with the recently updated BABAR and BELLE results. However, a value of $\sin 2 \beta \leq 0.2$, advocated by Silva and Wolfenstein as a benchmark for new physics, would suggest a violation in the three generation unitarity and would hint towards the existence of a fourth generation. Further, the future refinements in the CKM elements will push the lower limit on $\sin 2 \beta$ still higher.
\end{abstract}

PACS 11.30.Er, 12.15.Hh, 13.25.Hw

Keywords CKM matrix, unitarity, CP violating phase, reference triangle

The recent measurements of the time dependent CP asymmetry $a_{\psi K_{S}}$ in $B_{d}^{o}\left(\bar{B}_{d}^{o}\right) \rightarrow$ $\psi K_{S}$ decay by BABAR and BELLE collaborations, for example,

$$
\begin{array}{lll}
a_{\psi K_{S}}=0.12 \pm 0.37 \pm 0.09 & & \text { BABAR [1], } \\
a_{\psi K_{S}}=0.45_{-0.44}^{+0.43+0.09} & & \text { BELLE [2], }
\end{array}
$$


look to be smaller compared to the CDF measurements [3], for example,

$$
a_{\psi K_{S}}^{\mathrm{CDF}}=0.79_{-0.44}^{+0.41},
$$

as well as compared to the recent standard analysis of the unitarity triangle [4] with $\left|\epsilon_{K}\right|,\left|\frac{V_{u b}}{V_{c b}}\right|, \Delta m_{d}$ and $\Delta m_{s}$ as input, given as

$$
a_{\psi K_{S}}^{\mathrm{SM}}=0.67 \pm 0.17 .
$$

This disagreement gets reduced in the recently updated measurements of BABAR and BELLE, for example,

$$
\begin{array}{lll}
a_{\psi K_{S}}=0.34 \pm 0.20 \pm 0.05 & & \text { BABAR [5], } \\
a_{\psi K_{S}}=0.58_{-0.34}^{+0.32}{ }_{-0.10}^{+0.09} & & \text { BELLE [6], }
\end{array}
$$

however, the possibility of the $a_{\psi K_{S}}$ being lower than the predictions of Standard Model (SM) is still not ruled out. In the SM, $a_{\psi K_{S}}$ is related to the angle $\beta$ of the unitarity triangle as,

$$
a_{\psi K_{S}}=\operatorname{Sin} 2 \beta .
$$

Recently, several authors [7] - [9] have explored the implications of the possibility of low value of $\sin 2 \beta$ in comparison to the CDF measurements as well as to the global analysis of the unitarity triangle. These analyses lead to the general consensus that the possibility of new physics could be more prominent in the loop dominated processes, in particular the $B^{o}-\bar{B}^{o}$ mixing. Further, it is realized that the new physics will not affect the tree level decay processes and the unitarity of the three generation CKM matrix in the SM approaches as well as in its extensions [7]- 14]. In this connection, for better appraisal of new physics, it has been generally recommended to construct a universal or reference unitarity triangle [7], [10]-[14], wherein the inputs are free from the processes which might include the new physics effects, in particular the $B^{o}-\bar{B}^{o}$ mixing and $K^{o}-\bar{K}^{o}$ mixing parameters. Keeping this in mind several strategies, model dependent [10, 11] as well as model independent [12, 13, 14], have been formulated to construct the triangle, however by and large both approaches rely on the rare decays. The reference triangle to be constructed is defined as,

$$
V_{u d} V_{u b}^{*}+V_{c d} V_{c b}^{*}+V_{t d} V_{t b}^{*}=0,
$$

obtained by employing the orthogonality of the first and third column of the CKM matrix (henceforth referred to as triangle $d b$ ). In this triangle the elements involving $t$ quark have not been experimentally measured as yet and hence to construct the triangle, the inputs from rare decays involving elements $V_{t d}$ and $V_{t b}$ through loops have to be used. 
In this context, it is interesting to note that despite several analyses of the CKM phenomenology in the past [4], [15] - [18] yielding valuable information, the implications of three generation unitarity have not been examined in detail in the construction of the reference triangle. A reference triangle constructed purely from the considerations of unitarity as well as using experimentally measured CKM elements will be free from the effects of new physics and hence could serve as a tool for deciphering deviation from the SM in measuring the $\mathrm{CP}$ asymmetries.

The purpose of the present communication is to explore the construction of the triangle $d b$ using unitarity of the three generation CKM matrix as well as the existence of nonzero CP violating phase $\delta$, by evaluating the Jarlskog's Rephasing Invariant Parameter $J$ and consequently the $\delta$. In particular, we intend to evaluate angles $\alpha, \beta$ and $\gamma$ of the triangle $d b$ and study the implications of the low value of $\sin 2 \beta$ for unitarity.

To begin with, we consider the six non diagonal relations implied by the unitarity of the CKM matrix. One of the relations corresponds to equation 8 and the other five are as follows,

$$
\begin{aligned}
d s & V_{u d} V_{u s}^{*}+V_{c d} V_{c s}^{*}+V_{t d} V_{t s}^{*}=0, \\
s b & V_{u s} V_{u b}^{*}+V_{c s} V_{c b}^{*}+V_{t s} V_{t b}^{*}=0, \\
u t & V_{u d} V_{t d}^{*}+V_{u s} V_{t s}^{*}+V_{u b} V_{t b}^{*}=0, \\
u c & V_{u d} V_{c d}^{*}+V_{u s} V_{c s}^{*}+V_{u b} V_{c b}^{*}=0, \\
c t & V_{c d} V_{t d}^{*}+V_{c s} V_{t s}^{*}+V_{c b} V_{t b}^{*}=0 .
\end{aligned}
$$

The letters before the equations denote the respective triangles.

As mentioned above, in the triangle $d b$ the elements $V_{t d}$ and $V_{t b}$ are not experimentally measured, therefore the triangle cannot be constructed without additional inputs. This also corresponds to our ignorance of the $\mathrm{CP}$ violating phase $\delta$ of the CKM matrix, defined as 19

$$
V_{C K M}=\left(\begin{array}{lll}
c_{12} c_{13} & s_{12} c_{13} & s_{13} e^{-i \delta} \\
-s_{12} c_{23}-c_{12} s_{23} s_{13} e^{i \delta} & c_{12} c_{23}-s_{12} s_{23} s_{13} e^{i \delta} & s_{23} c_{13} \\
s_{12} s_{23}-c_{12} c_{23} s_{13} e^{i \delta} & -c_{12} s_{23}-s_{12} c_{23} s_{13} e^{i \delta} & c_{23} c_{13}
\end{array}\right)
$$

with $c_{i j}=\cos \theta_{i j}$ and $s_{i j}=\sin \theta_{i j}$ for $i, j=1,2,3$. In the above representation, the mixing angles $s_{12}, s_{23}$ and $s_{13}$ can be obtained from the experimentally well known elements $\left|V_{u s}\right|,\left|V_{c b}\right|$ and $\left|\frac{V_{u b}}{V_{c b}}\right|$ given in Table 1, hence the CP violating phase $\delta$ remains the only unknown parameter in determining the triangle $d b$. The phase $\delta$, however, is related to the Jarlskog's rephasing invariant parameter $J$ as

$$
J=s_{12} s_{23} s_{13} c_{12} c_{23} c_{13}^{2} \sin \delta
$$


Therefore, an evaluation of $J$ would allow us to find $\delta$ and consequently the angles $\alpha, \beta$ and $\gamma$ of the triangle $d b$. To evaluate $J$, we make use of the fact that the areas of all the six triangles (equations $8[13$ ) are equal and that the area of any of the unitarity triangle is related to Jarlskog's Rephasing Invariant Parameter $J$ as

$$
J=2 \times \text { Area of any of the Unitarity Triangle. }
$$

This, therefore affords an opportunity to evaluate $J$ through one of the unitarity triangle whose sides are experimentally well known, for example, triangle $u c$. The triangle $u c$ though is quite well known, but is highly squashed, therefore one needs to be careful while evaluating $J$ through this triangle. The sides of the triangle represented by $\left|V_{u d}^{*} V_{c d}\right|(=a)$ and $\left|V_{u s}^{*} V_{c s}\right|(=b)$ are of comparable lengths while the third side $\left|V_{u b}^{*} V_{c b}\right|(=c)$ is several orders of magnitude smaller compared to $a$ and $b$. This creates complications for evaluating the area of the triangle without violating the existence of $\mathrm{CP}$ violation. To avoid these complications, without violating the unitarity, we have incorporated the constraints $|a|+|c|>|b|$ and $|b|+|c|>|a|$ [20], this ensures that the triangle exists and has nonzero area. Using these constraints and the experimental data given in the table 1, a histogram can be plotted, shown in figure 1, to which a gaussian is fitted yielding the result,

$$
J=(2.59 \pm 0.79) \times 10^{-5} .
$$

Calculating $s_{12}, s_{23}$ and $s_{13}$ from the experimental values of $\left|V_{u s}\right|,\left|\frac{V_{u b}}{V_{c b}}\right|$, and $\left|V_{c b}\right|$ given in table 1, one can plot a distribution for $\delta$ as well using equation 15 and 17 and incorporating the above mentioned constraints. Again the distribution for $\delta$ is gaussian and yields,

$$
\delta=50^{\circ} \pm 20^{\circ}
$$

which in the second quadrant translates to,

$$
\delta=130^{\circ} \pm 20^{\circ} .
$$

This value of $\delta$ apparently looks to be the consequence only of the unitarity relationship given by equation 12. However on further investigations, as shown by Branco and Lavoura [20], one finds that this $\delta$ range is consequence of all the non trivial unitarity constraints. In this sense the above range could be attributed to unitarity of the CKM matrix. It needs to be noted that with the above range of $\delta$ and the experimental values of $\left|V_{u s}\right|,\left|V_{c b}\right|$ and $\left|\frac{V_{u b}}{V_{c b}}\right|$ given in Table 1, the CKM matrix thus evaluated is in excellent agreement with PDG 
CKM matrix [19]. In particular $\left|V_{t d}\right|$, the element with the most sensitive dependence on $\delta$ comes out to lie in the range,

$$
V_{t d}=0.0046 \text { to } 0.0134,
$$

in comparison with the PDG range, $V_{t d}=0.004$ to 0.014 .

In order to reinforce our conclusion regarding $J$, we have also evaluated $J$ through other unitarity triangles given in equations 8 to 13 . Without getting into the details, which will be published elsewhere, we have considered the evaluation of $J$ through the two unsquashed triangles $d b$ and $u t$. Since these triangles involve elements which are not experimentally known, for them we have used their PDG values based on the unitarity considerations. Following the procedure used for evaluating $J$ through triangle $u c$, we get the following values for $J$ evaluated through $d b$ and ut respectively,

$$
\begin{aligned}
& J=(2.51 \pm 0.87) \times 10^{-5} \\
& J=(2.45 \pm 0.91) \times 10^{-5}
\end{aligned}
$$

Quite interestingly, we find that $J$ values evaluated through these triangles are very much in agreement with the $J$ evaluated through the triangle $u c$. We, therefore, would like to emphasize that our evaluation of $J$ and $\delta$ from the squashed triangle $u c$ is very much consistent with the unitarity based evaluation of CKM matrix by PDG.

After having obtained $\delta$, the triangle $d b$ can be constructed, however without involving inputs from the phenomena which may have influence from the new physics as well as without the inputs from the rare decays. The angles $\alpha$, $\beta$ and $\gamma$ of the triangle can be expressed in terms of the CKM elements as,

$$
\begin{aligned}
& \alpha=\arg \left(\frac{-V_{t d} V_{t b}^{*}}{V_{u d} V_{u b}^{*}}\right), \\
& \beta=\arg \left(\frac{-V_{c d} V_{c b}^{*}}{V_{t d} V_{t b}^{*}}\right), \\
& \gamma=\arg \left(\frac{-V_{u d} V_{u b}^{*}}{V_{c d} V_{c b}^{*}}\right),
\end{aligned}
$$

where CKM elements are as given by the PDG representation in the equation 14. In Table 11 we have listed the experimental values of the CKM elements as given by PDG [19] as well as their future values. Making use of the PDG representation of CKM matrix given in equation 14, experimental values of $\left|V_{u s}\right|, \quad\left|V_{c b}\right|$ and $\left|\frac{V_{u b}}{V_{c b}}\right|$ from table 10 and $\delta$ given by equations 18 and 19, one can easily find out the corresponding ranges for the three angles. In the Table 
2, we have listed the corresponding results for $J, \delta, \alpha, \beta$ and $\gamma$. The ranges for $\alpha, \beta$ and $\gamma$ are as follows,

$$
\begin{aligned}
\alpha \simeq & 19^{\circ} \text { to } 139^{\circ}, \\
\beta \simeq & 6^{\circ} \text { to } 31^{\circ}, \\
\gamma \simeq & 30^{\circ} \text { to } 70^{\circ} \quad \text { in I quadrant, and } \\
& 110^{\circ} \text { to } 150^{\circ} \quad \text { in II quadrant. }
\end{aligned}
$$

While evaluating the three angles, we have taken care that the triangle is closed. The range of $\sin 2 \beta$ corresponding to equation 27 is given as,

$$
\sin 2 \beta=0.21 \text { to } 0.88 \text {. }
$$

It needs to be emphasized that this range for $\sin 2 \beta$ is obtained by making use of unitarity and the well known CKM elements listed in Table [1. The above range has considerable overlap with the BABAR and BELLE results, however if $\sin 2 \beta$ is found to be $\leq 0.2$, a benchmark for new physics as advocated by Silva and Wolfenstein [8], then one may conclude that even the three generation unitarity may not be valid and one may have to go to four generations to explain the low values of $\sin 2 \beta$. In such a scenario, the widely advocated assumption [7] - [14] that the non SM physics resides in loop dominated processes only may not be valid.

A few comments are in order. It needs to be pointed out that while evaluating the area of the unitarity triangle $u c$, we have assumed that $\mathrm{CP}$ violating phase $\delta$ is nonzero and that the triangle $u c$ exists and hence has nonzero area. In order to incorporate this in plotting the histogram we have not considered the entries corresponding to area of the triangle being zero and the case corresponding to one of the sides being larger than the sum of the other two sides. Both of these possibilities are not unambiguously ruled out by the present data because of the fact that the uncertainties in the two larger sides are greater than the third shorter side. However, the important point to be noted is the fact that our evaluation of $J$ and $\delta$ obtained by incorporating the above mentioned constraints does reproduce the PDG CKM elements based on the experimental input and unitarity.

It is interesting to examine the consequences of the future refinements in the CKM elements. While listing the future values of the elements we have considered only those elements where the present error is more than $15 \%$, for example $\left|\frac{V_{u b}}{V_{c b}}\right|$ and $\left|V_{c s}\right|$. The future values of these elements are listed in column III of Table 1 1 . One finds from the Table 2 that the refinements in $\left|\frac{V_{u b}}{V_{c b}}\right|$ and $\left|V_{c s}\right|$ would improve the lower bound on $\sin 2 \beta$ from 0.21 to 0.31 . This would give a clear signal for physics beyond the $\mathrm{SM}$ in case $\sin 2 \beta$ is measured to be $\leq 0.2$. To emphasize this conclusion, we have also considered all the future inputs at their $90 \%$ CL and this gives the lower limit of $\sin 2 \beta=0.18$. 
It may be of interest to mention that recent investigations involving texture 4 zeros quark mass matrices and unitarity [21], yield the following range for $\sin 2 \beta$,

$$
\operatorname{Sin} 2 \beta=0.27 \text { to } 0.60,
$$

which looks to be compatible with the present unitarity based calculations. A value of $\sin 2 \beta \leq 0.2$ therefore, will have far reaching consequences for unitarity as well as for texture specific mass matrices [21, 22, 23].

It is interesting to compare our results (equation 30) with those of Buras

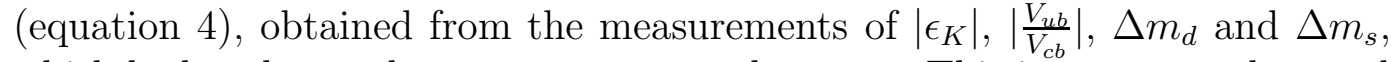
which look to be much narrower compared to ours. This is easy to understand when one considers the definition of $\beta$ given in equation 24, wherein the magnitude and phase of $V_{t d}$ play an important role. For example, the range of $\delta$ given by equation 18 and 19 yields the $V_{t d}$ range as 0.0046 to 0.0134 , whereas the range corresponding to Buras's analysis is 0.0067 to 0.0093 , which is narrower primarily due to restrictions imposed by $\left|\epsilon_{K}\right|, \Delta m_{d}$ and $\Delta m_{s}$. Our preliminary investigations wherein we have incorporated the constraints due to $\left|\epsilon_{K}\right|, \Delta m_{d}$ and $\Delta m_{s}$ along with the unitarity lead to results which are in agreements with those of Buras.

To conclude, we have explored the possibility of construction of a reference unitarity triangle by making use of the three generation unitarity of the CKM matrix, the existence of nonzero $\mathrm{CP}$ violating phase $\delta$ and the experimental values of the well known CKM elements, without involving any inputs from the processes which might include the new physics effects, in particular the $B^{o}-\bar{B}^{o}$ mixing and $K^{o}-\bar{K}^{o}$ mixing parameters as well as the rare decays. The angles of the triangle have been evaluated by finding the $\mathrm{CP}$ violating phase $\delta$ through the Jarlskog's rephasing invariant parameter $J$. Also, the $J$ and $\delta$ evaluated through the triangle $u c$ lead to the magnitudes of CKM matrix elements which are in full agreement with the PDG CKM matrix. The present data and the unitarity of the CKM matrix give $\delta=50^{\circ} \pm 20^{\circ}$, which translates to $130^{\circ} \pm 20^{\circ}$ in the second quadrant. The corresponding range for $\sin 2 \beta$ is 0.21 to 0.88 . This range is broadly in agreement with the recently updated BABAR and BELLE results and also has considerable overlap with the range found from the texture 4 zeros quark mass matrices and the unitarity of the CKM matrix. However, a value of $\sin 2 \beta \leq 0.2$ advocated by Silva and Wolfenstein as a benchmark for new physics would imply a violation in the three generation unitarity and would hint towards the existence of a fourth generation. Further, the future refinements in the CKM elements will push the lower limit on $\sin 2 \beta$ still higher, for example from 0.21 to 0.31 , thus giving a clear signal for physics beyond the $\mathrm{SM}$ in case $\sin 2 \beta$ is measured to be $\leq 0.2$. This remains valid even when the future values are considered at their $90 \%$ CL. 


\section{ACKNOWLEDGMENTS}

M.G. would like to thank S.D. Sharma for useful discussions. M.R. would like to thank CSIR, Govt. of India, for financial support and also the Chairman, Department of Physics, for providing facilities to work in the department.

\section{References}

[1] David G. Hitlin, BABAR Collaboration, hep-ex/0011024.

[2] Hiroaki Aihara, BELLE Collaboration, hep-ex/0010008.

[3] T. Affolder et al., CDF Collaboration, Phys. Rev. D61, 072005(2000).

[4] Andrzej J. Buras, hep-ph/0101336 and references therein.

[5] B. Aubert et al., BABAR Collaboration, hep-ph/0102030.

[6] A. Abashian et al., BELLE Collaboration, hep-ph/0102018.

[7] A. L. Kagan and M. Neubert, hep-ph/0007360.

[8] J. P. Silva and L. Wolfenstein, hep-ph/0008004.

[9] G. Eyal, Y. Nir and G. Perez, hep-ph/0008009; Z. Z. Xing, hepph/0008018; Y. Nir, hep-ph/0008226; A. J. Buras and R. Buras, hep$\mathrm{ph} / 0008273$.

[10] T. Goto, N. Kitazawa, Y. Okada and M. Tanaka, Phys. Rev. D53, 6662(1996).

[11] A. J. Buras, P. Gambino, M. Gorbahn, S. Jager and L. Silvestrini, hepph/0007085.

[12] Y. Grossmann, Y. Nir and M. P. Worah, Phys. Lett. B407, 307(1997).

[13] A. G. Cohen, D. B. Kaplan, F. Lepeintre, A. E. Nelson, Phys. Rev. Lett. $782300(1997)$.

[14] G. Barenboim, G. Eyal and Y. Nir, Phys. Rev. Lett. 83, 4486(1999).

[15] CP violation, Ed. L. Wolfenstein, North Holland, elsevier Science Publishers B.V., 1989; CP violation, Ed. C. Jarlskog, World Scientific Publishing Co. Pte. Ltd, 1989.

[16] Manmohan Gupta and P. S. Gill, Pramana 38, 477(1992); P. S. Gill and Manmohan Gupta, Mod. Phys. Lett. A13, 2445(1998). 
[17] L. Wolfenstein, Phys. Rev. Lett. 51, 1945(1983); Stefan Herrlich and Ulrich Nierste, Phys. Rev. D52, 6505(1995); M. Gronau and J. L. Rosner, Phys. Rev. Lett. 76, 1200(1996); H. Fritzsch and Z. Z. Xing, Nucl. Phys. B556, 49(1999); J.L. Rosner, hep-ph/0005258; A. Ali, Eur. Phys. J. C9, 687(1999); hep-ph/0002167; I. I. Bigi and A. I. Sanda, hep-ph/9909479;

R.D. Peccei, hep-ph/9904456; hep-ph/9909236; hep-ph/0004152.

[18] M. Ciuchini, G. D’Agostini, E. Franco, V. Lubicz, G. Martinelli, F. Parodi, P. Roudeau and A. Stocchi, hep-ph/0012308 and references therein.

[19] D.E. Groom et al., Particle Data group, Euro. Phys. J. C15, 1(2000).

[20] G.C. Branco and L. Lavoura, Phys. Lett. B208, 123(1988).

[21] Monika Randhawa and Manmohan Gupta, Phys. Rev. D63, 097301(2001).

[22] H. Fritzsch and Z.Z. Xing, Phys. Lett. B353, 114(1995); Nucl. Phys. B556, 49(1999); R. Barbieri, L.J. Hall and A. Romanino, Nucl. Phys. B551, 93(1999).

[23] T. Ito and M. Tanimoto, Phys. Rev D55, 1509(1997); T. Ito, N. Okamura and M. Tanimoto, Phys. Rev. D58, 077301(1998). 


\begin{tabular}{|l|l|l|}
\hline Parameter & PDG values [19] & Future values \\
\hline$\left|V_{u d}\right|$ & $0.9735 \pm 0.0008$ & $0.9735 \pm 0.0008$ \\
$\left|V_{u s}\right|$ & $0.2196 \pm 0.0023$ & $0.2196 \pm 0.0023$ \\
$\left|V_{c d}\right|$ & $0.224 \pm 0.016$ & $0.224 \pm 0.016$ \\
$\left|V_{c s}\right|$ & $1.04 \pm 0.16$ & $1.04 \pm 0.08$ \\
$\left|V_{c b}\right|$ & $0.0402 \pm 0.0019$ & $0.0402 \pm 0.0019$ \\
$\left|\frac{V_{u b}}{V_{c b}}\right|$ & $0.090 \pm 0.025$ & $0.090 \pm 0.010$ \\
\hline
\end{tabular}

Table 1: Values of the CKM parameters used throughout the paper.

\begin{tabular}{|c|c|c|c|}
\hline & With PDG values & With future values & $\begin{array}{c}\text { With future values } \\
\text { at their 90\% CL }\end{array}$ \\
\hline$J$ & $(2.59 \pm 0.79) \times 10^{-5}$ & $(2.79 \pm 0.49) \times 10^{-5}$ & $(2.61 \pm 0.78) \times 10^{-5}$ \\
$\delta$ & $50^{\circ} \pm 20^{\circ}$, & $60^{\circ} \pm 18^{\circ}$, & $55^{\circ} \pm 20^{\circ}$, \\
& $130^{\circ} \pm 20^{\circ}$ & $120^{\circ} \pm 18^{\circ}$ & $125^{\circ} \pm 20^{\circ}$ \\
$\alpha$ & $19^{\circ}$ to $141^{\circ}$ & $28^{\circ}$ to $124^{\circ}$ & $19^{\circ}$ to $143^{\circ}$ \\
$\beta$ & $6^{\circ}$ to $31^{\circ}$ & $9^{\circ}$ to $31^{\circ}$ & $5^{\circ}$ to $36^{\circ}$ \\
$\gamma$ & $50^{\circ} \pm 20^{\circ}$, & $60^{\circ} \pm 18^{\circ}$, & $55^{\circ} \pm 20^{\circ}$, \\
$\gamma$ & $130^{\circ} \pm 20^{\circ}$ & $120^{\circ} \pm 18^{\circ}$ & $125^{\circ} \pm 20^{\circ}$ \\
\hline
\end{tabular}

Table 2: $J, \delta$ and corresponding $\alpha, \beta$ and $\gamma$ with PDG and the future values of input parameters listed in Table 1 


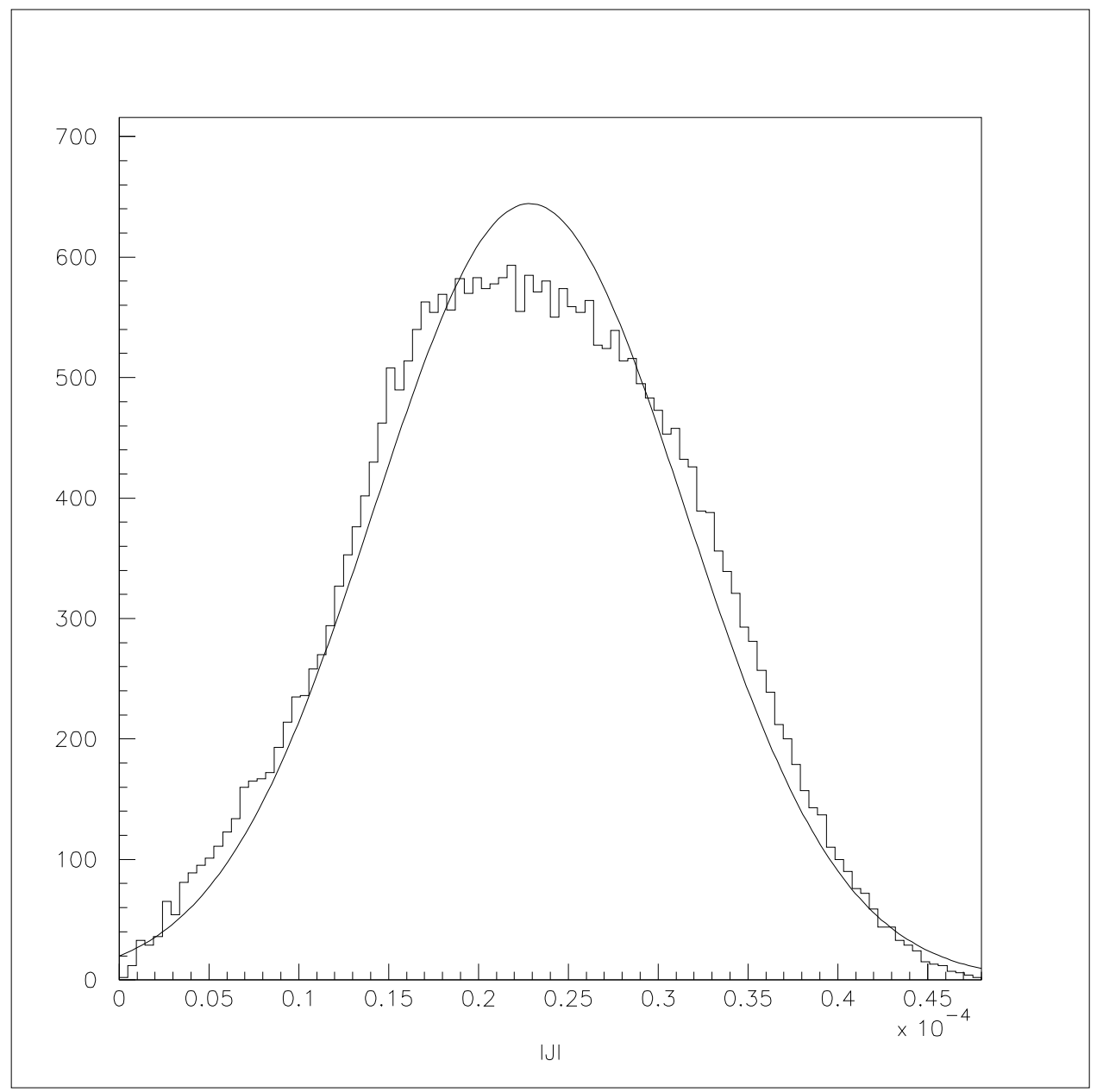

Figure 1: Gaussian fitted to the histogram of $|J|$ plotted by considering the triangle $u c$ with the input constraints $|a|+|c|>|b|$ and $|b|+|c|>|a|$, where $a=\left|V_{u d}^{*} V_{c d}\right|, b=\left|V_{u s}^{*} V_{c s}\right|$ and $c=\left|V_{u b}^{*} V_{c b}\right|$. 